\title{
Search for charged Higgs bosons in ATLAS using the $\tau+$ jets final state
}

\author{
Anna Kopp*, on behalf of the ATLAS Collaboration \\ Albert-Ludwigs-Universität Freiburg \\ E-mail: akopp@cern.ch
}

The results of searches for charged Higgs bosons decaying to a $\tau$ lepton and a neutrino are presented. These searches use $19.5 \mathrm{fb}^{-1}$ of proton-proton collision data at $\sqrt{s}=8 \mathrm{TeV}$ taken by the ATLAS experiment at the LHC, and consider cases where charged Higgs bosons are lighter or heavier than the top quark. Only final states with hadronically decaying $\tau$ leptons and additional jets from a hadronically decaying $W$ boson are taken into account. No excess of data over the expected Standard Model background is observed. Limits are set on the product of branching ratios $\mathscr{B}\left(t \rightarrow H^{+} b\right) \times \mathscr{B}\left(H^{+} \rightarrow \tau^{+} v\right)$ for charged Higgs bosons lighter than the top quark and on the production cross section times branching ratio $\sigma\left(p p \rightarrow \bar{t} H^{+} X\right) \times \mathscr{B}\left(H^{+} \rightarrow \tau^{+} v\right)$ for charged Higgs bosons that are heavier than the top quark.

Prospects for Charged Higgs Discovery at Colliders

16-18 September 2014

Uppsala University Sweden

\footnotetext{
* Speaker.
} 


\section{Introduction}

Charged Higgs bosons $\left(H^{+}, H^{-}\right)^{1}$ are predicted by many extensions of the Standard Model (SM), e.g. supersymmetric ones such as the Minimal Supersymmetric Standard Model (MSSM) [1]. Since no charged scalar particle is present in the SM, the observation of charged Higgs bosons would be a clear indicator for new physics. In scenarios such as the MSSM, the main production mode for charged Higgs bosons at the LHC is connected to top quarks but depends on the mass of the $H^{+}\left(m_{H^{+}}\right)$. For charged Higgs boson masses smaller than the top quark mass $\left(m_{\text {top }}\right)$ the main $H^{+}$production mode is through top quark decays, $t \rightarrow H^{+} b$. If $m_{H^{+}}$is greater than $m_{\text {top }}$, charged Higgs bosons are produced in association with a top quark. The leading order production mechanisms are shown in Fig. 1 for $m_{H^{+}}<m_{\text {top }}$ on the left hand side and for $m_{H^{+}}>m_{\text {top }}$ in the center and right hand side plots. In the MSSM, the decay $H^{+} \rightarrow \tau^{+} v$ is dominant if the $H^{+}$is lighter than
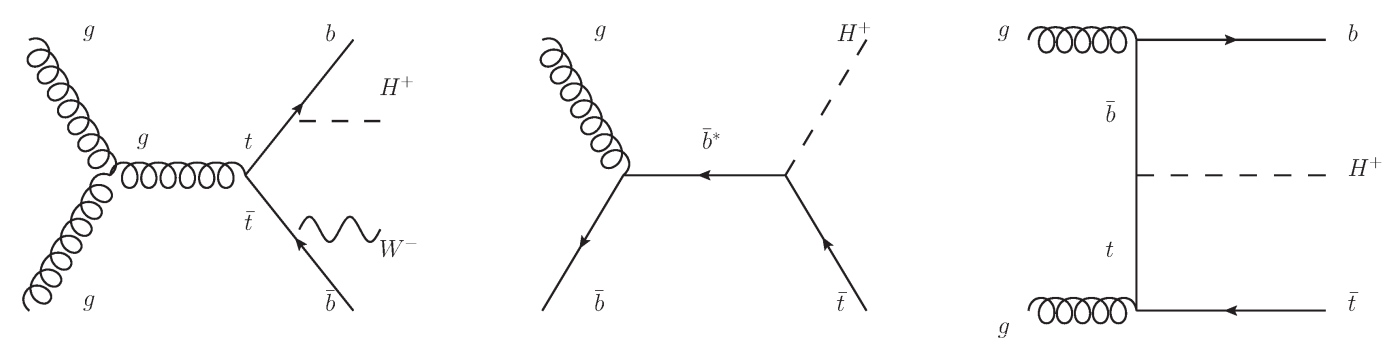

Figure 1: Leading-order Feynman diagrams for the dominant production modes of charged Higgs bosons at masses below (left) and above (center and right) the top quark mass.

the top quark for $\tan \beta>2$, where $\tan \beta$ is the ratio of the vacuum expectation values of the two Higgs doublets. This decay remains sizeable for $1<\tan \beta<2$. For $m_{H^{+}}>m_{\text {top }}$, the decay to a $\tau$ lepton and neutrino is still significant, especially for large values of $\tan \beta$.

In the analysis presented here, charged Higgs bosons are searched for in two mass ranges, $80 \mathrm{GeV}<$ $m_{H^{+}}<160 \mathrm{GeV}$ (low mass) and $180 \mathrm{GeV}<m_{H^{+}}<1000 \mathrm{GeV}$ (high mass). The mass region around the top quark mass is not taken into account because there are currently no reliable theoretical calculations available for this region. The final states considered in these searches exploit the presence of $H^{+} \rightarrow \tau^{+} v$ where the $\tau$ lepton decays hadronically, without the presence of any other charged lepton. The searches are model-independent. Thus, in the low mass search, exclusion limits are set on the branching ratio $\mathscr{B}\left(t \rightarrow H^{+} b\right) \times \mathscr{B}\left(H^{+} \rightarrow \tau^{+} v\right)$. Exclusion limits in the high mass search are given in terms of the production cross section $\sigma\left(p p \rightarrow \bar{t} H^{+} X\right) \times \mathscr{B}\left(H^{+} \rightarrow \tau^{+} v\right)$. The results are based on $19.5 \mathrm{fb}^{-1}$ of proton-proton collision data at $\sqrt{s}=8 \mathrm{TeV}$ collected by the ATLAS experiment [2] at the Large Hadron Collider and presented in Ref. [3].

\section{Event selection}

The final state topology in the searches presented here is characterized by a hadronically decaying $\tau$ lepton, missing transverse momentum, jets including $b$-tagged jets and no electrons or muons.

\footnotetext{
${ }^{1}$ In the following, charged Higgs bosons are denoted by $\mathrm{H}^{+}$and the charge-conjugate is implied.
} 
Both the low and high mass $H^{+}$search are based on events passing a $\tau+E_{\mathrm{T}}^{\text {miss }}$ trigger, where $E_{\mathrm{T}}^{\text {miss }}$ is the magnitude of the missing transverse momentum. The threshold on the $p_{\mathrm{T}}$ of the $\tau$ trigger object started at $27 \mathrm{GeV}$ and was increased to $29 \mathrm{GeV}$, while the threshold for $E_{\mathrm{T}}^{\text {miss }}$ started at $45 \mathrm{GeV}$ and was increased to $50 \mathrm{GeV}$. The following requirements are applied in the selection:

- at least 4 (3) jets with $p_{\mathrm{T}}>25 \mathrm{GeV}$ in the low (high) mass $H^{+}$search

- at least one of these jets has to be $b$-tagged

- exactly one hadronically decaying $\tau$ lepton with $p_{\mathrm{T}}>40 \mathrm{GeV}$ that is matched to the $\tau$ trigger object

- no isolated electron or muon with high transverse momentum

- $E_{\mathrm{T}}^{\text {miss }}>65(80) \mathrm{GeV}$ in the low (high) mass $H^{+}$search

- $\frac{E_{\mathrm{T}}^{\text {miss }}}{\sqrt{\Sigma p_{\mathrm{T}}^{\mathrm{PVtrk}}}}>6.5(6) \mathrm{GeV}^{1 / 2}$ in the low (high) mass $H^{+}$search, where $\Sigma p_{\mathrm{T}}^{\mathrm{PV} \text { trk }}$ is the sum of transverse momenta of all tracks originating in the primary vertex.

The transverse mass $m_{\mathrm{T}}$, given by

$$
m_{\mathrm{T}}=\sqrt{2 p_{\mathrm{T}}^{\tau} E_{\mathrm{T}}^{\mathrm{miss}}\left(1-\cos \Delta \phi_{\tau, \mathrm{miss}}\right)},
$$

is used as the final discriminant. Here, $\Delta \phi_{\tau \text {,miss }}$ is the azimuthal angle between the hadronic decay products of the $\tau$ lepton and the direction of the missing transverse momentum. A requirement of $m_{\mathrm{T}}>20(40) \mathrm{GeV}$ is placed on events passing the low (high) mass signal selection. This cut is motivated in Section 3.1.

\section{Background estimation}

Background events in the analyses presented here are classified by the origin of the $\tau$ lepton in the event. Data-driven methods are used to estimate backgrounds with true $\tau$ leptons and jets that are misidentified as $\tau$ leptons. Background events where an electron or a muon is misidentified as a $\tau$ lepton are estimated from simulation.

\subsection{Background events with true hadronically decaying $\tau$ leptons}

An embedding method is used to estimate the background contribution with true hadronically decaying $\tau$ leptons. A control sample of muon+jets events is selected using similar criteria as for the default event selection except for any $\tau$ lepton related requirements. The muon signature is then extracted, removed from the event and replaced by the signature of a hadronically decaying $\tau$ lepton from simulation. These new events are then used for the background prediction. An advantage of this method compared to using simulation is that almost everything in the final event, including contributions from pile-up, the underlying event and missing transverse momentum, except for the neutrino from the $\tau$ lepton decay, is taken directly from data. Additionally, the method does not rely on theoretical cross sections and their uncertainties since the normalization of the background is determined from data, only assuming lepton universality in the $W$ boson decay.

The following criteria are applied to select the muon+jets sample: 
- a single muon trigger

- exactly one isolated muon with $p_{\mathrm{T}}>25 \mathrm{GeV}$

- no isolated electron with $p_{\mathrm{T}}>20 \mathrm{GeV}$

- at least 4 (3) jets with $p_{\mathrm{T}}>25 \mathrm{GeV}$ for the low (high) mass $H^{+}$search

- at least one of these selected jets is $b$-tagged

- $E_{\mathrm{T}}^{\text {miss }}>25(40) \mathrm{GeV}$ for the low (high) mass $H^{+}$search

Although these selections are looser than the $\tau+$ jets signal selections used in this analysis, a bias is introduced by the $E_{\mathrm{T}}^{\text {miss }}$ cut. This results in missing events in the very low $m_{\mathrm{T}}$ bins. Thus a cut on $m_{\mathrm{T}}>20(40) \mathrm{GeV}$ is introduced in the low (high) mass search to remove the bias.

To replace a muon with a simulated $\tau$ lepton, the track of the selected muon in the data is extracted and removed. Any energy deposited in the calorimeter is removed by simulating a $W \rightarrow$ $\mu \nu$ decay with the same kinematics as in the selected data event. Calorimeter cells identified as corresponding to that decay are then removed from the original event. To account for the higher $\tau$ lepton mass, the momentum of the original muon is rescaled,

$$
\vec{p}_{\tau}=\frac{\sqrt{E_{\mu}^{2}-m_{\tau}^{2}}}{\sqrt{\vec{p}_{\mu} \cdot \vec{p}_{\mu}}} \vec{p}_{\mu} .
$$

Here, $\vec{p}_{\tau}$ is the rescaled momentum, $\vec{p}_{\mu}$ is the reconstructed muon momentum, $E_{\mu}$ is the reconstructed muon energy and $m_{\tau}$ is the $\tau$ lepton mass. The $\tau$ lepton is then processed by TAUOLA [4] and PHOTOS to produce a hadronic $\tau$ lepton decay as well as to account for polarization and final state radiation. The decay products are propagated through full detector simulation and reconstruction. The original event without the muon and the hadronic $\tau$ lepton decay products are then merged to the final embedded event.

The full signal selection is then applied to the sample of embedded events to derive the shape of the $m_{\mathrm{T}}$ distribution. Furthermore, the normalization is taken directly from the number of embedded events:

$$
N_{\tau}=N_{\text {embedded }} \cdot\left(1-c_{\tau \rightarrow \mu}\right) \frac{\varepsilon^{\tau+E_{\mathrm{T}}^{\text {miss }} \text {-trigger }}}{\varepsilon^{\mu-\text { ID,trigger }}} \times \mathscr{B}(\tau \rightarrow \text { hadrons }+v),
$$

where $N_{\text {embedded }}$ is the number of embedded events that pass the signal selection, $c_{\tau \rightarrow \mu}$ is a correction factor to account for incorrectly embedded $W \rightarrow \tau \rightarrow \mu$ decays, $\varepsilon^{\tau+E_{\mathrm{T}}^{\text {miss }}-\text { trigger }}$ are the $\tau+E_{\mathrm{T}}^{\text {miss }}$ trigger efficiencies applied as functions of $p_{\mathrm{T}}$ of the $\tau$ lepton and $E_{\mathrm{T}}^{\text {miss }}, \varepsilon^{\mu-\mathrm{ID} \text {,trigger }}$ represent muon trigger and identification efficiencies, applied as functions of $p_{\mathrm{T}}$ and $\eta$ of the original muon and $\mathscr{B}(\tau \rightarrow$ hadrons $+v)$ is the branching ratio of $\tau$ lepton decays to hadrons.

The fully normalized $m_{\mathrm{T}}$ distribution resulting from the embedding method is shown in Fig. 2 and compared to simulation.

\subsection{Multi-jet background}

A matrix method is used to estimate the background contribution where a jet is misidentified as a hadronically decaying $\tau$ lepton. Two data samples are defined that differ only in the $\tau$ identification criteria: a loose and a tight sample, where the tight sample is enriched in true hadronically 


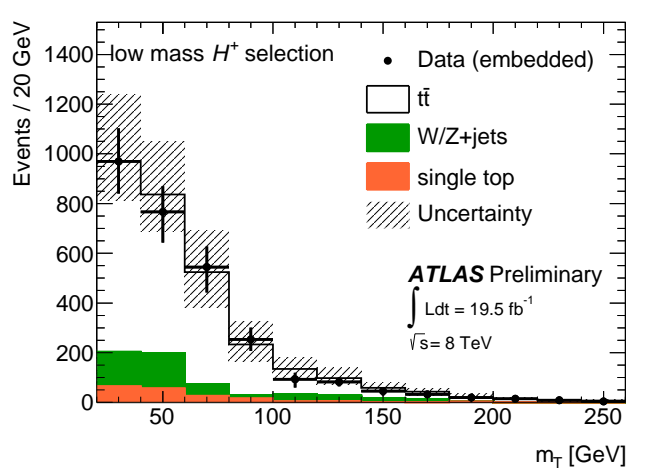

(a) Low mass $H^{+}$selection

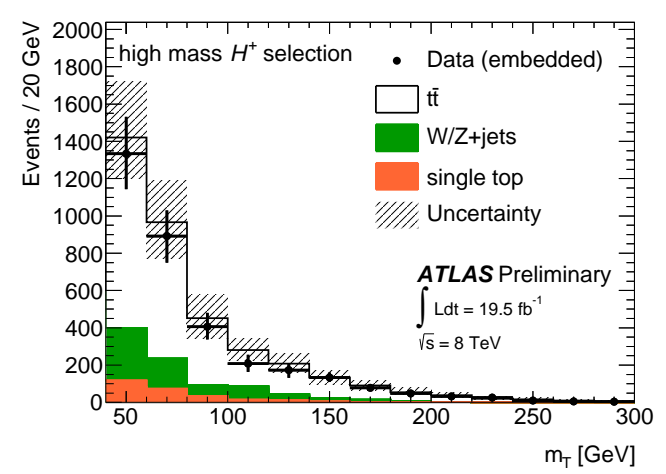

(b) High mass $H^{+}$selection

Figure 2: Comparison of the $m_{\mathrm{T}}$ distributions for events with a true hadronically decaying $\tau$ lepton for the low mass (a) and high mass (b) charged Higgs boson search, as predicted by the embedding method and simulation. Combined statistical and systematic uncertainties for the embedded sample are shown as error bars, and all systematic uncertainties applicable to simulation are shown as hatched bands. Taken from Ref. [3]

decaying $\tau$ leptons compared to the loose sample and corresponds to the signal region. By construction the tight sample is a sub-set of the loose sample. The loose sample contains $N_{\mathrm{r}}$ and $N_{\mathrm{m}}$ events with real and misidentified hadronically decaying $\tau$ leptons, respectively. It also consists of a certain number $N_{\mathrm{L}}$ events that pass loose but not tight $\tau$ identification and a number $N_{\mathrm{T}}$ that pass the tight selection. If the efficiencies $p_{\mathrm{m}}$ and $p_{\mathrm{r}}$ are defined as the probabilities for real or misidentified hadronically decaying $\tau$ leptons that pass loose identification to also pass tight identification, the following relation can be established:

$$
\left(\begin{array}{c}
N_{\mathrm{T}} \\
N_{\mathrm{L}}
\end{array}\right)=\left(\begin{array}{cc}
p_{\mathrm{r}} & p_{\mathrm{m}} \\
\left(1-p_{\mathrm{r}}\right) & \left(1-p_{\mathrm{m}}\right)
\end{array}\right) \times\left(\begin{array}{c}
N_{\mathrm{r}} \\
N_{\mathrm{m}}
\end{array}\right) .
$$

After inverting the matrix above, the number of events with misidentified hadronically decaying $\tau$ leptons passing the tight selection, $N_{\mathrm{m}}^{\mathrm{T}}$ is given by:

$$
N_{\mathrm{m}}^{\mathrm{T}}=p_{\mathrm{m}} N_{\mathrm{m}}=\frac{p_{\mathrm{m}} p_{\mathrm{r}}}{p_{\mathrm{r}}-p_{\mathrm{m}}} N_{\mathrm{L}}+\frac{p_{\mathrm{m}}\left(p_{\mathrm{r}}-1\right)}{p_{\mathrm{r}}-p_{\mathrm{m}}} N_{\mathrm{T}}
$$

The efficiencies $p_{\mathrm{r}}$ and $p_{\mathrm{m}}$ are parametrized in the number of charged particle tracks in a cone with $\Delta R<0.2$ and in a hollow isolation cone with $0.2<\Delta R<0.4$ around the axis of the hadronically decaying $\tau$ lepton, as well as in the transverse momentum and $\eta$ of the hadronically decaying $\tau$ lepton. Finally, weights are computed based on $p_{\mathrm{r}}$ and $p_{\mathrm{m}}$ and applied to events in the loose sample to estimate the number of events with misidentified hadronically decaying $\tau$ leptons in the tight sample:

- for an event with a loose but not tight hadronically decaying $\tau$ lepton, $w_{\mathrm{L}}=\frac{p_{\mathrm{m}} p_{\mathrm{r}}}{p_{\mathrm{r}}-p_{\mathrm{m}}}$;

- for an event with a tight hadronically decaying $\tau$ lepton, $w_{\mathrm{T}}=\frac{p_{\mathrm{m}}\left(p_{\mathrm{r}}-1\right)}{p_{\mathrm{r}}-p_{\mathrm{m}}}$. 
The probability $p_{\mathrm{m}}$ is measured in a $W+$ jets control region in data and $p_{\mathrm{r}}$ is estimated using simulated $t \bar{t}$ events in the signal region.

Events with jets misidentified as hadronically decaying $\tau$ leptons are a major background in the high $m_{\mathrm{T}}$ region, but this region is statistically limited. To circumvent this limitation, the $m_{\mathrm{T}}$ distribution is fit using a power log function $f(x)=x^{a+b \ln (x)}$, in the mass range $200-800 \mathrm{GeV}$. The fits for the low and high mass $H^{+}$search, the systematic uncertainty due to the choice of the fit function and the fits showing all other systematic uncertainties are shown in Fig. 3.

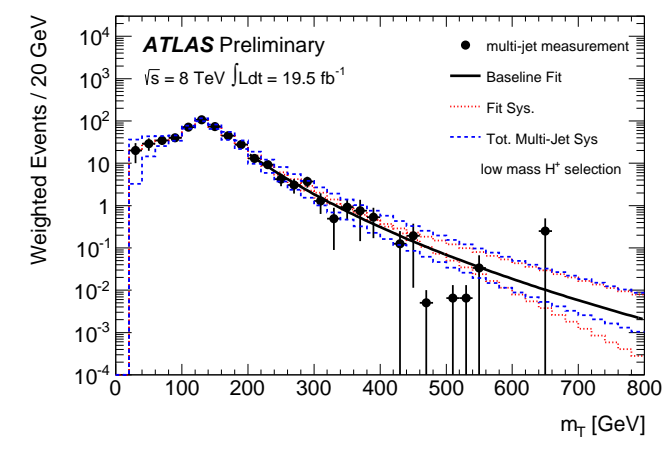

(a) Low mass $H^{+}$selection

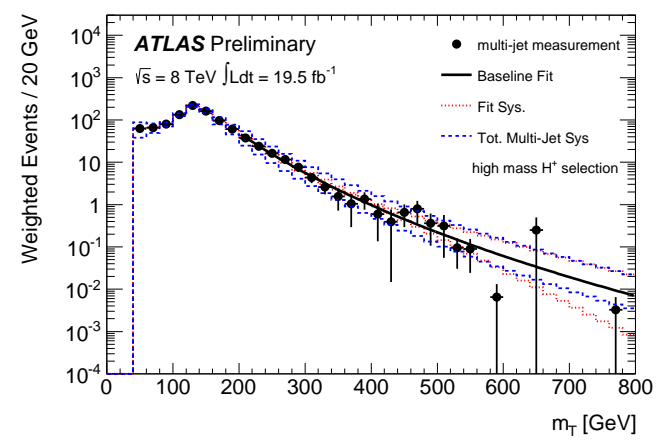

(b) High mass $H^{+}$selection

Figure 3: The multi-jet backgrounds for the (a) low and (b) high mass $H^{+}$selections, where the results of fits using a power log function are shown in black. The red dotted lines show the systematic uncertainty from the choice of the fit function. The blue dashed lines show the total combined fits from all sources of systematic uncertainties. Taken from Ref. [3]

\subsection{Background events with electrons or muons misidentified as hadronically decaying $\tau$ leptons}

Background events, where an electron or muon is misidentified as a hadronically decaying $\tau$ lepton are estimated based on simulation. Dedicated veto algorithms suppress these backgrounds heavily so that they contribute only at the order of $1-2 \%$ to the total background. They include contributions from $t \bar{t}, W+$ jets, $Z+$ jets and single top quark processes.

\section{4 $\tau+E_{\mathrm{T}}^{\text {miss }}$ triggers}

The event selections used in the analyses presented here rely on $\tau+E_{\mathrm{T}}^{\text {miss }}$ triggers. To correct for differences between measured trigger rates in data and simulation, correction factors are derived. They are evaluated depending on $p_{\mathrm{T}}^{\tau}$ and $E_{\mathrm{T}}^{\text {miss }}$ separately to increase the samples that are available and any residual correlations are taken into account as systematic uncertainties. A $\mu+\tau$ sample is selected in a control region enriched in $t \bar{t}$ events and a tag-and-probe method with a single muon trigger is used to determine the efficiencies. The $\tau$ and $E_{\mathrm{T}}^{\text {miss }}$ trigger efficiencies are then fit separately for $\tau$ leptons with 1 or 3 charged tracks in both data and simulation. The ratios of these fitted functions are used as continuous correction factors applied to simulation. They are shown in Fig. 4. Additionally, the efficiencies measured in data are used to normalize the embedded sample. No trigger information is available after embedding. The efficiencies derived from the $\mu+\tau$ sample need to be corrected for the high amount of objects misidentified as hadronically decaying $\tau$ 
leptons present in this sample when applied to the embedded sample, where only true hadronically decaying $\tau$ leptons are included.

\section{Systematic Uncertainties}

\section{1 $\tau+E_{\mathrm{T}}^{\text {miss }}$ triggers}

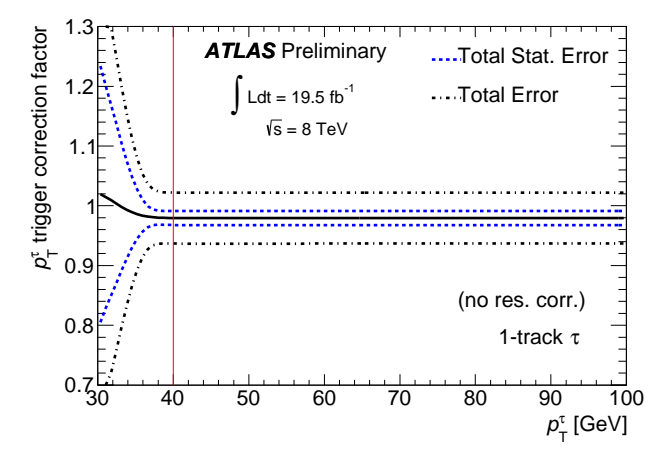

(a) 1-track $\tau, \tau$ trigger

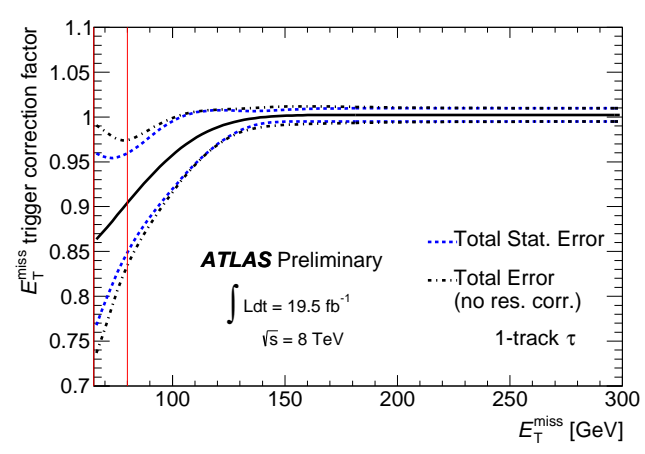

(c) 1 -track $\tau, E_{\mathrm{T}}^{\text {miss }}$ trigger

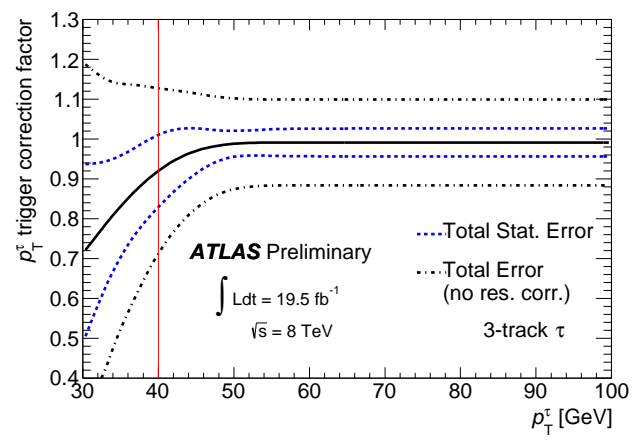

(b) 3-track $\tau, \tau$ trigger

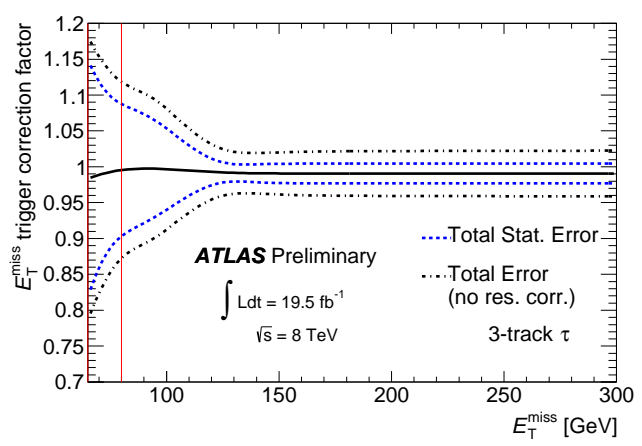

(d) 3-track $\tau, E_{\mathrm{T}}^{\text {miss }}$ trigger

Figure 4: Inclusive $\tau$ and $E_{\mathrm{T}}^{\text {miss }}$ trigger correction factors derived from the ratios of functions fitted to data and simulation for hadronically decaying $\tau$ leptons with one (three) charged tracks in the left (right) columns are shown at the top and bottom, respectively. The vertical line in Figure (a) and (b) indicates the lowest $p_{\mathrm{T}}^{\tau}$ threshold used in the analyses. The vertical line in Figures (c) and (d) shows the lower boundary of the high mass charged Higgs boson search. The total statistical uncertainty is indicated with the blue dotted line and the systematic uncertainty is added in quadrature to the statistical error (dashed-dotted line). Taken from Ref. [3].

The systematic uncertainty on the measurement of the $\tau+E_{\mathrm{T}}^{\text {miss }}$ trigger efficiencies arise from various sources: the selection of the muon in the $\mu+\tau$ sample, the amount of misidentified hadronically decaying $\tau$ leptons, the choice of the fitting function and slightly varying trigger requirements throughout the data-taking period as well as a residual correlation between the $\tau$ and $E_{\mathrm{T}}^{\text {miss }}$ triggers contribute to the overall systematic uncertainty. The effects on a low and high mass signal sample are shown in Table 1.

The trigger correction factors applied to simulation to account for differences in the measured efficiencies between data and simulation are shown in Figure 4. 


\begin{tabular}{l|c|c} 
Source of uncertainty & Low mass $\mathrm{H}^{+}$selection & High mass $\mathrm{H}^{+}$selection \\
\hline \hline Muon selection & $<1 \%$ & $<1 \%$ \\
Misidentified $\tau$ & $5.6 \%$ & $5.7 \%$ \\
Fitting function & $2.1 \%$ & $1.8 \%$ \\
Trigger definition & $<1 \%$ & $<1 \%$ \\
Residual correlations & $1.4 \%$ & $3.2 \%$ \\
$\tau$ energy scale & $<1 \%$ & $<1 \%$
\end{tabular}

Table 1: Effect of systematic uncertainties on the combined trigger efficiencies for a low mass $\left(m_{H^{+}}=\right.$ $130 \mathrm{GeV})$ and high mass $\left(m_{H^{+}}=250 \mathrm{GeV}\right)$ signal sample. Taken from Ref. [3]

\begin{tabular}{l|c|c} 
Source of uncertainty & Low mass $H^{+}$selection & High mass $H^{+}$selection \\
\hline \hline True $\tau$ & \multicolumn{2}{|c}{} \\
\hline Embedding parameters & $3.0 \%$ & $1.8 \%$ \\
Muon isolation & $0.3 \%$ & $2.3 \%$ \\
Parameters in normalization & $2.0 \%$ & $2.0 \%$ \\
$\tau$ identification & $2.2 \%$ & $2.0 \%$ \\
$\tau$ energy scale & $4.0 \%$ & $3.6 \%$ \\
$\tau+E_{\mathrm{T}}^{\text {miss }}$ trigger & $8.3 \%$ & $8.3 \%$ \\
\hline \hline Jet $\rightarrow \tau$ & \multicolumn{2}{|}{} \\
\hline Statistical uncertainty on $p_{\mathrm{m}}$ & $2.0 \%$ & $3.4 \%$ \\
Statistical uncertainty on $p_{\mathrm{r}}$ & $0.5 \%$ & $0.5 \%$ \\
Jet composition & $1.1 \%$ & $1.9 \%$ \\
$\tau$ identification & $0.8 \%$ & $0.6 \%$ \\
$e / \mu$ contamination & $0.5 \%$ & $0.7 \%$
\end{tabular}

Table 2: Dominant systematic uncertainties on the backgrounds estimated using data-driven methods. The shift in event yield is given relative to the total background. Taken from Ref. [3]

\subsection{Data-driven background estimation}

The embedding method used for estimating background events with true hadronically decaying $\tau$ leptons can introduce a potential bias when removing calorimeter deposits of the muon in the original event. Thus a systematic uncertainty is evaluated where the amount of energy that is subtracted is varied. A systematic uncertainty due to the muon isolation criteria used in the original $\mu+$ jets selection is evaluated by loosening and tighten these criteria. Any systematic uncertainties on the trigger efficiency measurement also affects the background with true hadronically decaying $\tau$ leptons. Since the $\tau$ lepton in the embedded event is taken from simulation, systematic uncertainties related to the $\tau$ energy scale and identification have to be taken into account as well.

For the background with jets that are misidentified as hadronically decaying $\tau$ leptons, the dominant systematic uncertainties are due to the statistical uncertainties related to the size of the control sample and signal regions. An uncertainty arising from different jet compositions in the 
control and signal regions is evaluted by measuring $p_{\mathrm{m}}$ in a control region that is enriched in gluoninitiated jets and comparing that value to the one measured in a control region enriched in quarkinitiated jets. Additional uncertainties are due to the uncertainty in the identification of simulated true $\tau$ leptons, the statistical uncertainty on $p_{\mathrm{r}}$ and the effect of the efficiency of the simulated electron veto for true electrons in the measurement of $p_{\mathrm{m}}$.

All systematic uncertainties on data-driven background estimation are summarized in Table 2.

\section{Results}

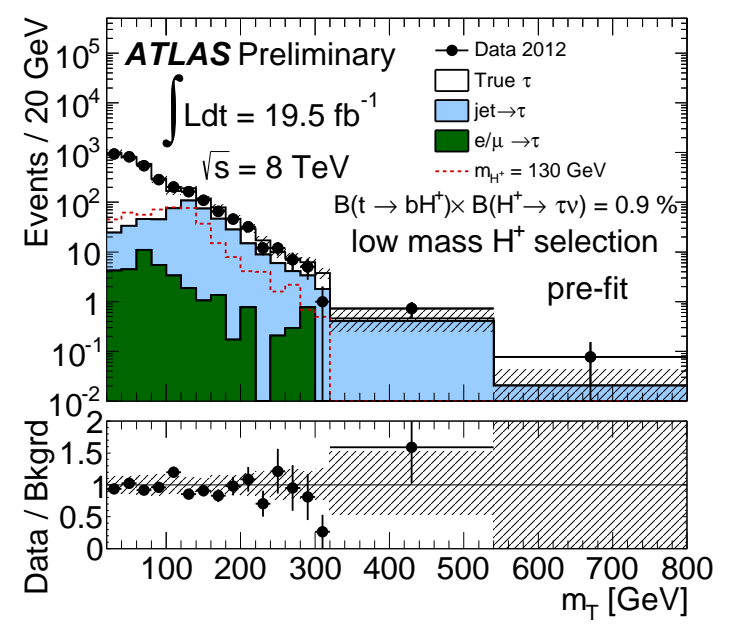

(a) Low mass $H^{+}$selection

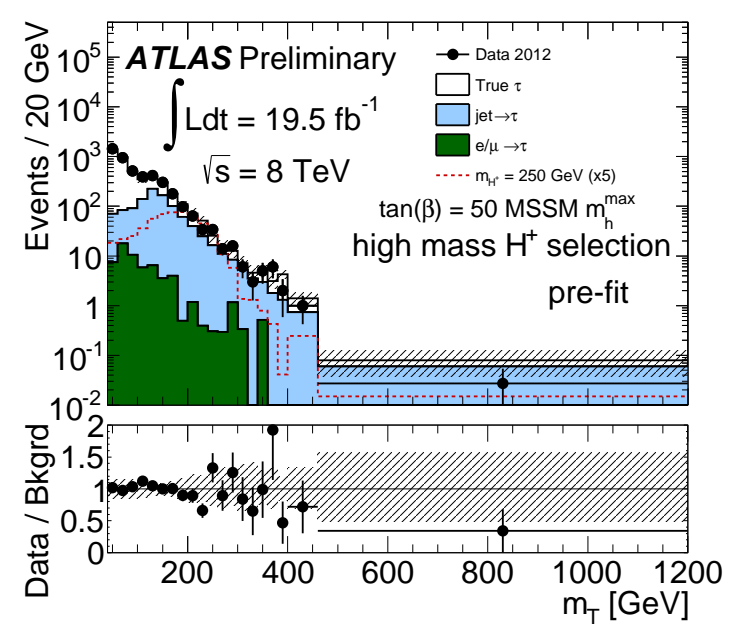

(b) High mass $H^{+}$selection

Figure 5: Distributions of $m_{\mathrm{T}}$ after all selection criteria and before the final fit. The hatched area shows the total pre-fit uncertainty for the SM backgrounds. For the low mass selection (a), bins are $20 \mathrm{GeV}$ wide up to $m_{\mathrm{T}}=320 \mathrm{GeV}$, then $320-540 \mathrm{GeV}$ and $>540 \mathrm{GeV}$. For the high mass selection (b), bins are $20 \mathrm{GeV}$ wide up to $m_{\mathrm{T}}=400 \mathrm{GeV}$ then $400-460 \mathrm{GeV}$ and $>460 \mathrm{GeV}$. All bins are normalized to $20 \mathrm{GeV}$ bin width. For the low mass search (a), a possible signal contribution with $m_{H^{+}}=130, \mathrm{GeV}$, and $\mathscr{B}\left(t \rightarrow b H^{+}\right) \times \mathscr{B}\left(H^{+} \rightarrow \tau^{+} v\right)=0.9 \%$ is stacked on top of the SM contributions. For the high mass search (b), a possible signal contribution with $m_{H^{+}}=250 \mathrm{GeV}$ and $\tan \beta=50$ in the $m_{h}^{\max }$ scenario of the MSSM, where the corresponding cross section is scaled up by a factor of 5, is stacked on top of the SM contributions. Taken from Ref. [3]

In Fig. 5 the final $m_{\mathrm{T}}$ distributions for the low and high mass $H^{+}$search are shown. No significant deviation between SM prediction and data is observed. Thus for the low mass charged Higgs boson search, exclusion limits are set on the branching ratio $\mathscr{B}\left(t \rightarrow H^{+} b\right) \times \mathscr{B}\left(H^{+} \rightarrow \tau^{+} v\right)$. For the high mass $H^{+}$search, limits are set on $\sigma\left(p p \rightarrow \bar{t} H^{+} X\right) \times \mathscr{B}\left(H^{+} \rightarrow \tau^{+} v\right)$, where the charge conjugate final state is included. Profile log-likelihood ratios [5] are used with $m_{\mathrm{T}}$ as discriminating variable to quantify the compatibility of the data with background-only and signal+background hypotheses. Exclusion limits are set by rejecting the signal hypothesis at the $95 \%$ confidence level using the $\mathrm{CL}_{s}$ procedure [6]. The resulting exclusion limits are shown in Fig. 6. 


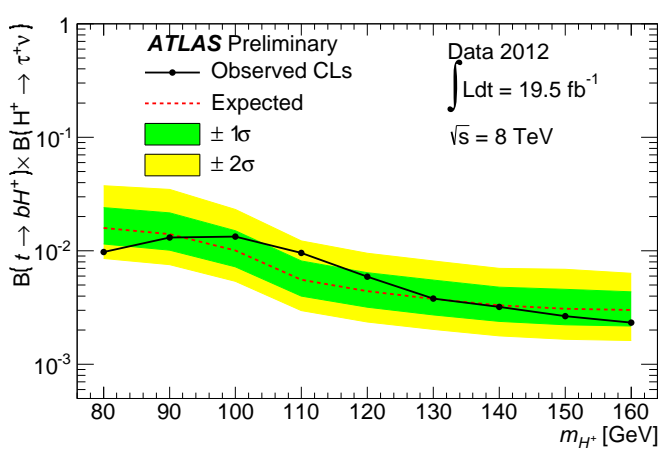

(a) Low mass $H^{+}$selection

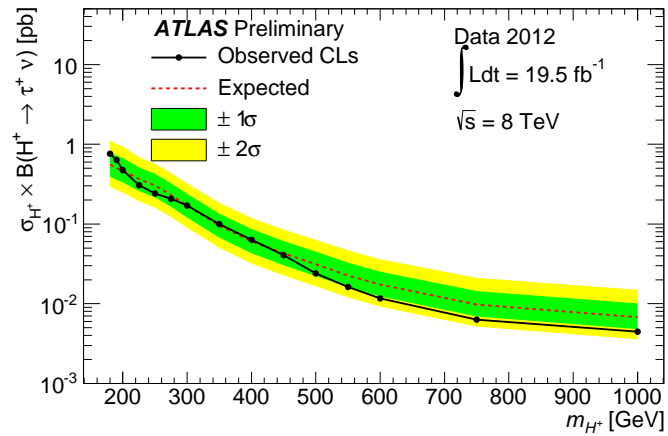

(b) High mass $H^{+}$selection

Figure 6: Observed and expected 95\% CL exclusion limits on the production and decay of low and high mass charged Higgs bosons. For the low mass search, the limit is set on $\mathscr{B}\left(t \rightarrow b H^{+}\right) \times \mathscr{B}\left(H^{+} \rightarrow \tau^{+} v\right)$ for Higgs boson production from top-quark decays as a function of $m_{H^{+}}$. For the high mass search, the limit is set on $\sigma\left(p p \rightarrow \bar{t} H^{+} X\right) \times \mathscr{B}\left(H^{+} \rightarrow \tau^{+} v\right)$, and are to be understood as applying to the total production cross section times branching ratio of $H^{+}$and $H^{-}$combined. Taken from Ref. [3]

\section{Conclusions}

Charged Higgs bosons are searched for either produced in top quark decays, $t \rightarrow H^{+} b$ (low mass) or in association with a top quark $\sigma\left(p p \rightarrow \bar{t} H^{+} X\right)$ (high mass), where $H^{+} \rightarrow \tau^{+} v$. For this purpose, a total of $19.5 \mathrm{fb}^{-1}$ of pp collision data at $\sqrt{s}=8 \mathrm{TeV}$ recorded by the ATLAS experiment in 2012 are analyzed. No significant deviation is observed between SM prediction and the data. Thus upper limits at the $95 \%$ confidence level are set on $\mathscr{B}\left(t \rightarrow H^{+} b\right) \times \mathscr{B}\left(H^{+} \rightarrow \tau^{+} v\right)$ between 0.2 and $1.3 \%$ in the mass range $80<m_{H^{+}}<160 \mathrm{GeV}$ in the low mass search and on $\sigma(p p \rightarrow$ $\left.\bar{t} H^{+} X\right) \times \mathscr{B}\left(H^{+} \rightarrow \tau^{+} v\right)$ between $0.76 \mathrm{pb}$ and $4.5 \mathrm{fb}$ in the mass range $180<m_{H^{+}}<1000 \mathrm{GeV}$.

\section{References}

[1] Lee, T.D., A Theory of Spontaneous T Violation, Phys.Rev. D8 (1973), 1226-1239.

[2] ATLAS Collaboration, The ATLAS Experiment at the CERN Large Hadron Collider, JINST3 (2008) S08003.

[3] ATLAS Collaboration, Search for charged Higgs bosons decaying via $H^{ \pm} \rightarrow \tau^{ \pm} v$ in hadronic final states using $p p$ collision data at $\sqrt{s}=8 \mathrm{TeV}$ with the ATLAS detector, ATLAS-CONF2014-050, https://cds.cern.ch/record/1756361.

[4] Was, Z. and Golonka, P., TAUOLA as tau Monte Carlo for future applications, Nucl. Phys. Proc. Suppl. 144 (2005) 88.

[5] Cowan, G., Cranmer, K., Gross, E. and Vitells, O. Asymptotic formulae for likelihood-based test of new physics, Eur. Phys. J. C. 71 (2011) 1554 [arXiv:1007.1727]

[6] Read, A. L., Presentation of search results: The CLs technique, J. Phys. G 28 (2002) 2693. 